\title{
Optimal siting and sizing of distributed energy storage systems via alternating direction method of multipliers
}

\author{
Mostafa Nick*, Rachid Cherkaoui, Mario Paolone \\ Distributed Electrical Systems Laboratory (DESL), École Polytechnique Fédérale de Lausanne, Lausanne, Switzerland
}

\section{A R T I C L E I N F O}

\section{Article history:}

Received 1 February 2015

Accepted 16 February 2015

Available online 9 March 2015

\section{Keywords:}

Alternating direction method of multipliers Active distribution networks

Energy storage

Optimal planning

Ancillary services

\begin{abstract}
A B S T R A C T
Energy Storage Systems (ESSs) will have an important role in the optimal operation of Active Distribution Networks (ADNs). Within this context, this paper focuses on the problem of ESSs optimal siting and sizing. Following similar approaches already proposed by the Authors, this paper proposes a multi-objective procedure that accounts for various ancillary services that can be provided by ESSs to ADNs. The proposed procedure takes into account the voltage support and network losses minimization along with minimization of the cost of energy from external grid and congestion management. For the case of large-scale problems, accounting for networks with large number of nodes and scenarios, the selection of the solution methodology is a non-trivial problem. In this respect, the paper proposes and discusses the use of the Alternative Direction Method of Multipliers in order to define an efficient algorithm capable to treat large-scale networks and, also, address the issue of the optimality of the solution. A real large-scale network with real profiles of load and distributed photovoltaic generation is used as the case study to analyze the effectiveness of the proposed methodology.
\end{abstract}

(c) 2015 Elsevier Ltd. All rights reserved.

\section{Introduction}

Active Distribution Networks (ADNs) are changing significantly by integrating new technologies aiming at improving their level of control. Energy Storage Systems (ESSs) have an important role in this context [1]. Indeed, they have the ability to be indirectly used to control the network providing several services like load shaving, supplementing renewable resources, and, as a consequence, postpone investments needed for network reinforcements (e.g., [2,3]). They are also capable of providing network ancillary services like support to voltage and frequency controls and indirect control line congestions. Additionally, they can be also used for network losses reduction [4-6]. In this respect, one of the main problems associated to the use of ESSs in ADNs is to find their best location and size (i.e., power and energy ratings) in order to maximize their impact on the grid.

In this context, several works have been done related to optimal planning of ESSs in ADNs. This issue has been addressed in both microgrids and ADNs. The Authors of [7] have proposed the use

\footnotetext{
This work is supported by the project between the EOS Holding and the EPFL Distributed Electrical Systems Laboratory entitled "Advanced control of distribution networks with the integration of dispersed energy storage systems".

* Corresponding author. Tel.: +4121 6934810; fax: +41216934662.

E-mail addresses: mostafa.nick@epfl.ch (M. Nick), rachid.cherkaoui@epfl.ch (R. Cherkaoui), mario.paolone@epfl.ch (M. Paolone).
}

of a Genetic Algorithm (GA) to find the optimal capacities of ESSs with the objective to minimize the operation costs of the targeted microgrid. A methodology to site and size different types of ESSs within the microgrid context has been proposed in [8]. A GA is used to find the best solution to maximize the total net present value. A methodology for optimal siting and sizing of ESSs in a medium voltage distribution network, with the goal of decreasing wind energy curtailment and minimizing annual cost of the electricity, is presented in [9]. A hybrid GA, sequential quadratic programming algorithm is proposed in [10] to size and site DGs, energy storage and reactive power compensation systems. The goals of the planning problem are the minimization of the total network losses and the operation costs. The Authors of [6] have presented a hybrid method integrating dynamic programming with GA to find the best siting, rating and control strategy of ESSs, in order to minimize the overall investments and network costs (energy cost and losses). A cost-benefit analysis methodology is presented in [11] to find the best sizing and siting of ESSs in distribution networks. The goal of the optimization is to maximize the Distribution Network Operator (DNO) profits from energy transactions, investment and operation cost savings. The planning of ESSs connected to transmission networks is also investigated in the literature (e.g., $[12,13])$. In [12] the optimal planning of ESSs in a network with renewable, and consequent uncertain energy production, is presented. The objective of the optimization is to minimize the 


\section{Nomenclature}

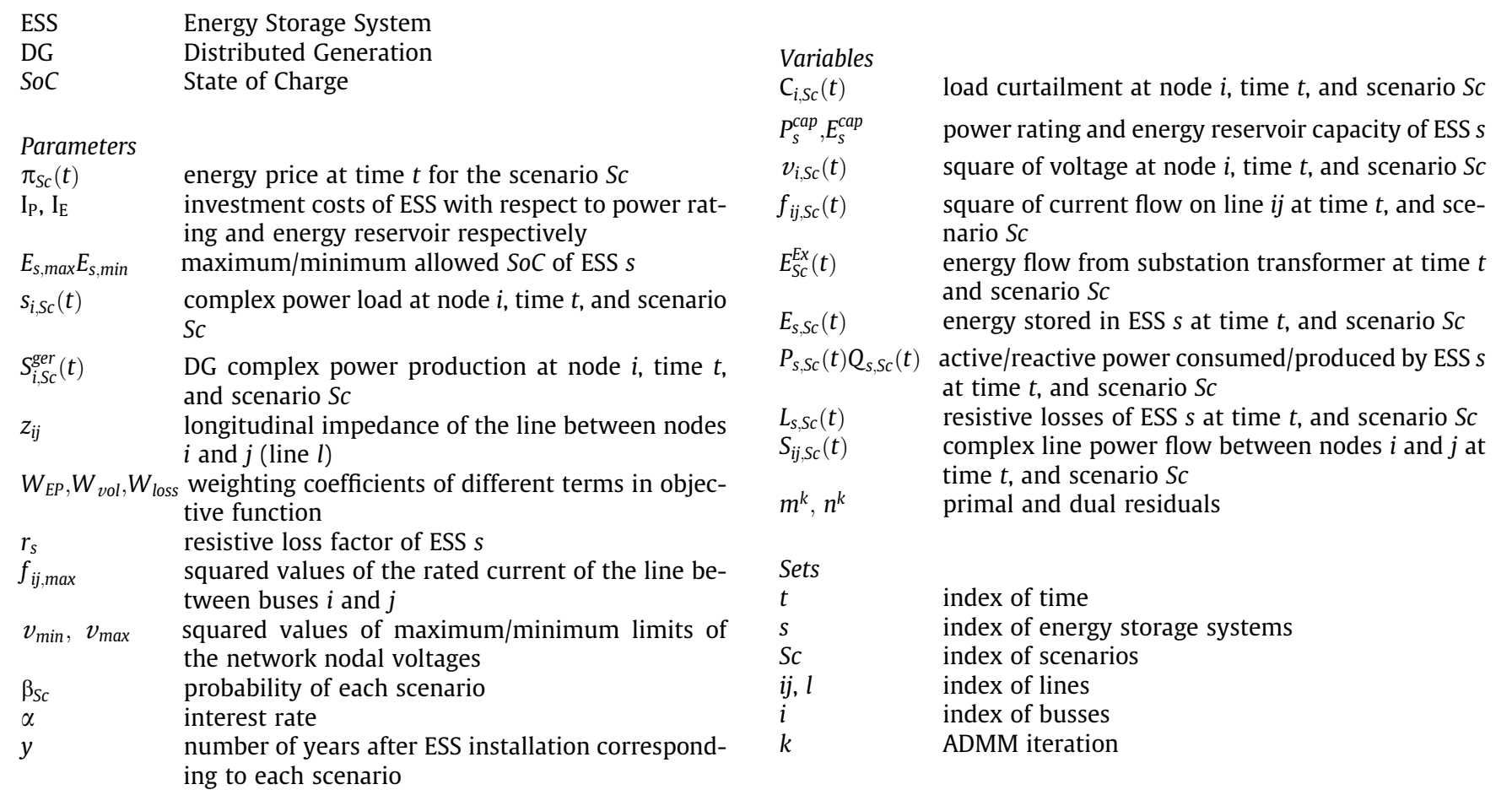

operation and investment costs of energy storage devices. The application of ESSs in optimal allocation of wind capacity related to distant wind farms is investigated in [13]. The methodology simultaneously optimizes the wind power capacity of each site, its ESS and the required transmission connection capacity.

A limitation of the above-listed papers is represented by the fact that they have not accounted in the problem formulation the ancillary services (e.g., voltage control) that ESSs can provide to ADNs. In this respect, the Authors of this paper have proposed in [4] a specific algorithm for assessing the optimal siting of ESSs to maximize their contribution to voltage control. Voltage sensitivity coefficients, as a function of the nodal power injections, were used to linearize the objective function of the problem and some of the constraints. The augmented problem of optimal allocation of ESSs in ADNs with a multi-objective (i.e., loss, energy cost, and voltage deviation minimizations) has been investigated in [14] by using a hybrid approach of GA and non-linear programming. Although the approach proposed in [14] provides satisfactory results, it is computationally expensive and the global optimal solution is not guaranteed due to the non-convexity of the problem. As a matter of fact, the computational inefficiency of this approach resulted into limiting the possibility of solving large-scale problems associated to: (i) networks with large number of nodes and (ii) multiple scenarios related to load and renewable resources volatilities (i.e., seasonal variability and yearly evolutions). In [15] a Second Order Cone Programming (SOCP) formulation of the optimal power flow (OPF) is used to define the problem of the optimal siting and sizing of ESSs in ADNs. It considers both technical and economical goals. However, as expected, the size of the problem increases drastically with the increase of both network size and number of scenarios. As a consequence, a dedicated decomposition method might be required. These drawbacks have motivated this contribution. Indeed, long-term optimal planning problems are normally largescale ones since they should include a reasonable number of scenarios to address the variations and uncertainties of various parameters. Decomposition methods can be used to breakdown large-scale problems into smaller ones. They have been used for several power system problems and typical application examples can be found in these references [16,17].

The purpose of the methodology presented in this paper is to provide a tool to the ADN operator's capable to define the optimal planning of their own energy storage systems to support the network operation in presence of massive stochastic distributed generation. In this respect, it is important to underline that the paper does not focus on the comparative assessment of different technical solutions to support to the ADN operation. In this work we propose to use the Alternating Direction Method of Multipliers (ADMM) $[18,19]$ to break down the original problem and have a distributed parallel convex optimization. The Second Order Cone programming (SOCP) OPF approach of [20] is adapted to formulate the problem of the optimal siting and sizing of ESSs in ADNs in order to obtain a convex problem. In this respect, it is worth mentioning that the convex formulation of ESSs planning is a peculiar aspect of the problem that has not been sufficiently addressed in the literature. The proposed approach also accounts for a non-simplified power flow in which the reactive power associated to shunt admittances of lines/cables is represented. Additionally, the ESSs are accurately modeled in terms of efficiency and SoC. Also their interfaces to the AC grid are represented by means of active and reactive power capability limits. The targeted problem is formulated as a multi-objective one including voltage deviations, network losses, in addition to investment and operation cost minimizations. It should be finally noted that this paper is an extended version of [21] which has been presented at the 18th Power System Computation Conference and was invited to be published in this journal.

The rest of the paper is organized as follows: the section 'Problem description' illustrates the problem and provides its formulation. The section 'Solution methodology' explains the proposed methodology to breakdown and solve the problem. The 
application example, referring to a real network configuration with real data, is presented in section 'Simulation results'. In order to highlight the peculiar capability of the proposed approach to deal with large-scale problems, section 'Computational performances' provides the analysis of the computation performances of the proposed algorithm with and without using the ADMM decomposition. The section 'Conclusion' closes the paper with final remarks concerning the applicability of the proposed procedure.

\section{Problem description}

The context of the problem refers to an ADN in presence of nondispatchable DGs and uncontrollable loads (i.e., we do not consider the presence of demand-side management). The objective is to find the best locations and sizes of a limited amount of ESSs where the limitation applies to the total DNO ESS investment. As anticipated, the problem accounts for two main objectives; (i) minimization of the investment costs associated to the installation of ESSs, (ii) minimization of a virtual cost that accounts for the network operation including both technical and economical costs. It should be noted that the charge/discharge cycles and the SoC level of ESSs are not considered in the operation costs. This is because these costs have been indirectly included in the problem by considering the ESSs limited lifetime (i.e., 5 years). The objective function is as in (1). It includes the investment cost of the ESSs and the network operation costs. The objective of the operation cost considers different terms: (i) voltage deviations from the rated value, (ii) cost of energy imported from the external grid, (iii) total network losses, and (iv) total load curtailment and (v) feeders congestions. The constraints of the problem are modeled by (2)-(12).

$$
\begin{aligned}
& \text { minimize : Investment cost }+ \text { Operation cost } \\
& \text { Investment cost }\left.=W_{E P}\left\{\left(P_{s}^{c a p} I_{P}\right)+\left(E_{s}^{c a p} I_{E}\right)\right)\right\} \\
& \text { Operation cost }= \frac{\beta_{S c}}{(1+\alpha)^{(y-1)}}\left(\sum _ { S c } \left[\sum _ { t } \left\{\sum _ { i } \left(W_{v o l}\left|v_{i, S c}(t)-1\right|:\left(v_{i, S c}(t)\right.\right.\right.\right.\right. \\
& \geqslant\left.\left.v_{t h r}^{\max }|| v_{i, S c}(t) \leqslant v_{t h r}^{\min }\right)+C_{i, S c}(t)\right) \\
&\left.\left.\left.+\sum_{l}\left(W_{\text {loss }} r_{l} f_{i, j, S c}(t):\left(f_{i j, S c}(t) \geqslant f_{t h r}\right)\right)+W_{E P} E_{S c}^{E x}(t) \pi_{S C}(t)\right]\right\}\right)
\end{aligned}
$$

Investment cost $\leqslant$ Total budget

$$
\begin{aligned}
& E_{s, S c}(t+1)=E_{s, S c}(t)-P_{s, S c}(t)-L_{s, S c}(t) \forall s \in E S S \\
& E_{s, \min } E_{s}^{c a p} \leqslant E_{s, S c}(t) \leqslant E_{s, \max } E_{s}^{c a p} \forall s \in E S S \\
& 0 \leqslant P_{s, S c}(t) \leqslant P_{s}^{c a p} \\
& P_{s, S c}^{2}(t)+Q_{s, S c}^{2}(t) \leqslant\left(P_{s}^{c a p}\right)^{2} \quad \forall s \in E S S \\
& L_{s, S c}(t) \geqslant r_{s}\left(P_{s, S c}^{2}(t)+Q_{s, S c}^{2}(t)\right) \quad \forall s \in E S S \\
& S_{i j, S c}(t)=s_{i, S c}(t)+\sum_{h: h \rightarrow i}\left(S_{h i, S c}(t)-z_{h i} f_{h i, S c}(t)\right)-S_{i, S c}^{g e r}(t) \\
& \quad-C_{i, S c}(t) \forall(i, j) \in G \\
& f_{i j, S c}(t) \geqslant \frac{\left|S_{i j, S c}(t)\right|^{2}}{v_{i, S c}(t)} \forall(i, j) \in G \\
& f_{i j, S c}(t) \leqslant f_{i j, \max } \quad \forall(i, j) \in G \\
& v_{i, S c}(t)=v_{j, S c}(t)+2 \operatorname{Re}\left(\bar{z}_{i j} S_{i j, S c}(t)\right)-\left|z_{i j}\right|^{2} f_{i j, S c}(t) \\
& v_{\min } \leqslant v_{i, S c}(t) \leqslant v_{\max } i \in N
\end{aligned}
$$

The total budget constraint is represented by (2). The energy stored in each ESS is dependent on the previous SoC and the amount of energy stored/withdrawn from the ESS reservoir: Eq. (3) models this constraint. Eqs. (4) and (5) show the capacity constraints of ESSs power rating and energy reservoir. The capability curve of the ESSs is accounted by (6). It is worth observing that this constraint is piece-wise linearized in order to preserve the convexity of the whole optimization problem [15]. ${ }^{1}$ The resistive losses of each ESS, associated to its efficiency, are approximated by equation (7) where we have considered them proportional to the square of the apparent power flow flowing through a given ESS. Constraints (8)-(12) define the constraints associated to the network operation. The power balance is modeled by (8). The feeders current flow limits are modeled by $(9)^{2}$ and (10) and the voltage limits are defined by (11) and (12).

\section{Solution methodology}

Summary about the alternating direction method of multipliers [18]

The inherent large-scale nature of the problem lays in the fact that it should cover a reasonable number of scenarios in order to obtain a solution accounting for a sufficiently large set of variations of the considered parameters. One of the most common approaches is to breakdown the problem into smaller ones. By following this idea, we propose to use the ADMM to breakdown the original problem and obtain a distributed parallel convex optimization ones.

The ADMM is a powerful and well-suited method for decentralized convex optimization. The peculiarity of the ADMM is that it uses a decomposition-coordination procedure in order to find the solution to a large global problem by solving small local subproblems in parallel. It uses the benefits of dual decomposition and augmented Lagrangian methods $[18,19]$. In the following, the ADMM is briefly described.

Suppose an optimization problem generically represented by (13) and (14) where $f, g$, $\xi$, and $\zeta$ are convex. The $f$ and $g$ are independent from each other except they are linked by the constraint (14).

$$
\begin{array}{ll}
\text { minimize }_{x, z} & f(x)+g(z) \\
\text { subject to } & \operatorname{dom} f=\{x \mid x \in \xi\} \\
& \operatorname{dom} g=\{z \mid z \in \zeta\}
\end{array}
$$

$A x+B z=c$

where $A \in \mathbb{R}^{p \times n}, B \in \mathbb{R}^{p \times m}$ and $c \in \mathbb{R}^{p}$ when the variables $x \in \mathbb{R}^{n}$ and $z \in \mathbb{R}^{m}$. The augmented Lagrangian of this problem with respect to constraint (14) has the form (15).

$$
\begin{aligned}
& L_{\rho}(x, y, z)=f(x)+g(z)+y^{T}(A x+B z-c)+(\rho / 2)\|A x+B z-c\|_{2}^{2} \\
& \text { subject to } \operatorname{dom} f=\{x \mid x \in \xi\} \\
& \qquad \operatorname{dom} g=\{z \mid z \in \zeta\}
\end{aligned}
$$

The ADMM procedure is deployed as follows. First, the augmented Lagrangian problem (15) is minimized with $z$ and $y$ being fixed. Then the obtained $x$ is used in the minimization of (15) with $x$, and $y$ being fixed. Finally, the dual multipliers are updated as shown in (18) with the obtained $x$ and $z$ from the previous step. This procedure will be continued until it converges to the global optimal solution.

\footnotetext{
1 The linearization of the constraints of the problem has been already addressed by the Authors in [15].

2 This constraint is the relaxed version of its original formulation composed by an equality instead of an inequality.
} 
$x^{k+1}:=\operatorname{argmin}_{x} L_{\rho}\left(x, y^{k}, z^{k}\right)$

$z^{k+1}:=\operatorname{argmin}_{z} L_{\rho}\left(x^{k+1}, y^{k}, z\right)$

$y^{k+1}:=y^{k}+\rho\left(A x^{k+1}+B z^{k+1}-c\right)$

\section{ADMM application to ESSs optimal planning}

The ADMM is used here to decompose the problem of the installation-cost minimization from the one of the operation-cost

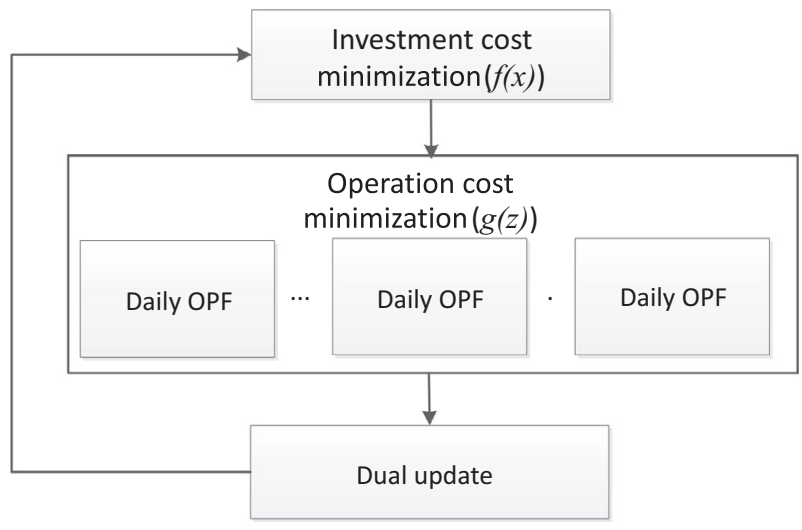

Fig. 1. ADMM procedure applied to the problem of ESS optimal planning. minimization enabling a parallel formulation. The two problems are linked by a set of linear constraints. These constraints imply that the ESS capacities obtained in the first problem are identical to the ones in the second. The application of the ADMM to the ESS optimal planning problem is described in what follows. Functions $f(x)$ and $g(z)$ of (13) represent, in our case, the ESS investment and the operation cost functions respectively. The linking constraints are the capacities (power rating and energy reservoir) of the ESSs in investment and operation cost minimizations. They assure that the ESSs power rating and energy reservoir capacities should be the same for the two ADMM sub-problems. The objective function to be minimized in the first step is the investment cost minimization of (1) and it is constrained by total budget. The operation cost of (1) is the objective that is minimized in the second step. The constraints of the second step are (3)-(12). The last step is the dual update. These steps are iterated until the solver converges to an optimal solution. The scheme of the proposed ADMM-based procedure is shown in Fig. 1.

\section{Simulation results}

A real distribution network located in the southwest of Switzerland has been used as a case study (see Fig. 2). The network contains 287 nodes and is characterized by non-negligible amount of PV installations with a total capacity of $5 \mathrm{MW}$. Nodes where PVs are connected are shown in Fig. 2 together with the obtained location of the ESSs inferred from the proposed

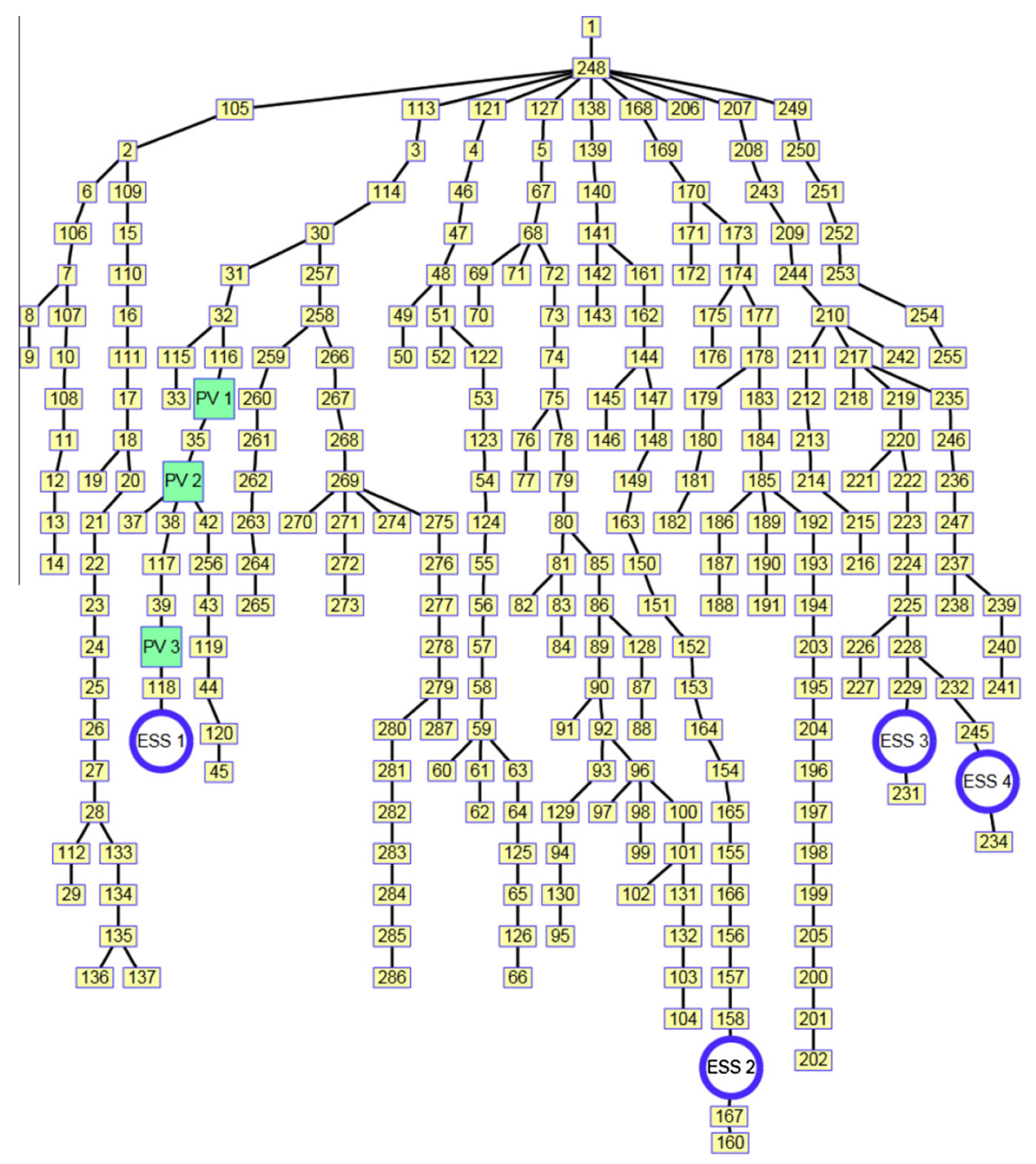

Fig. 2. The schematic of the test case study. 
procedure. The simulation is done for five years (assumed lifetime of ESSs) and four weeks in each year: one in spring, one in summer, one in fall, and one in winter. Experimentally measured loads and generation profiles for this specific grid are available with a discretization time step of $15 \mathrm{~min}$. Total active power load profiles of these four time periods for the first year are shown in Fig. 3. The load profiles for the other years are considered to have the same profile with a linear increase of $5 \%$ per year. The load is distributed between the feeders as shown in Table 1 . The energy price profiles of these weeks (for the first year) are shown in Fig. 4 . The energy price is assumed to increase by $2 \%$ at each year. The weight coefficients of the elements composing the objective function are: voltage deviation $W_{v o l}=0.61$, total network loss $W_{\text {loss }}=0.05$, energy cost from external grid $W_{e p}=0.04$ and the feeder overloading above $80 \%$ of their respective capacities $W_{v o l}=0.3$. These values have been inferred using the Analytic Hierarchy Process (AHP) [22]. First, the relative importance of each element of the multi->objective function is defined by the decision-maker (in this case, the DNO). Then, a pairwise comparison is done between the objectives. These pairwise comparisons are used to form a matrix. Finally, this matrix is used to calculate the final weights.

The investment costs for ESSs capacity rating and energy reservoir are assumed to be $1800 \mathrm{CHF} / \mathrm{kW}$ and $1000 \mathrm{CHF} / \mathrm{kWh}$ respectively. These values are inferred from the report [23] with specific reference to the Li-ion electrochemical batteries.

The annual interest rate is assumed to be $4 \%$. The ADMM penalty parameter $\rho$ has been assumed equal to 100 . The voltage minimization term in the objective function is activated when
Table 1

Average feeder loading in the four considered weeks. It should be noted that the feeder connecting the bus \#206 is not considered in this table.

\begin{tabular}{lllllllll}
\hline Feeder \# & 1 & 2 & 3 & 4 & 5 & 6 & 7 & 8 \\
\hline $\begin{array}{c}\text { Starting node of the } \\
\text { considered feeder } \\
\text { (see Fig. 2) }\end{array}$ & 105 & 113 & 121 & 127 & 138 & 168 & 207 & 249 \\
$\begin{array}{c}\text { Load share with } \\
\text { respect to total } \\
\text { network loading } \\
\text { (\%) }\end{array}$ & 0.62 & 25.1 & 13.2 & 7 & 20 & 5 & 0.29 & 28 \\
& & & & & & & & \\
\hline
\end{tabular}

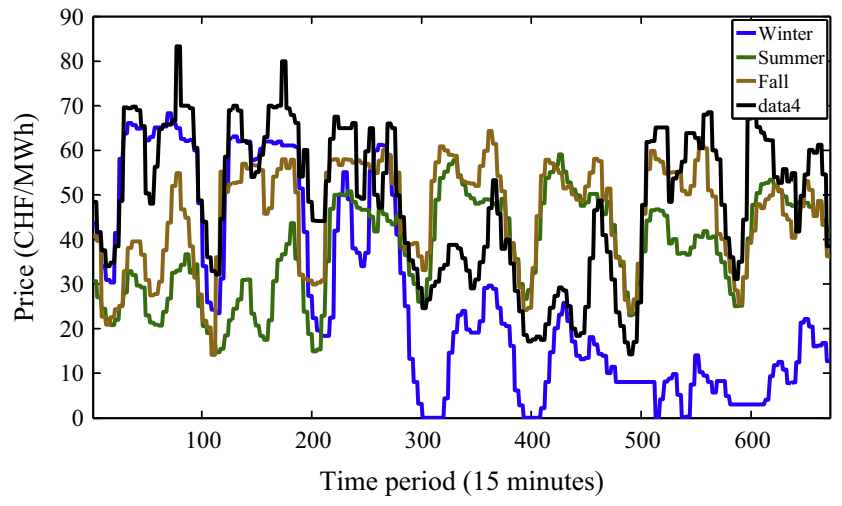

Fig. 4. Profiles of the electricity prices in the four considered weeks.
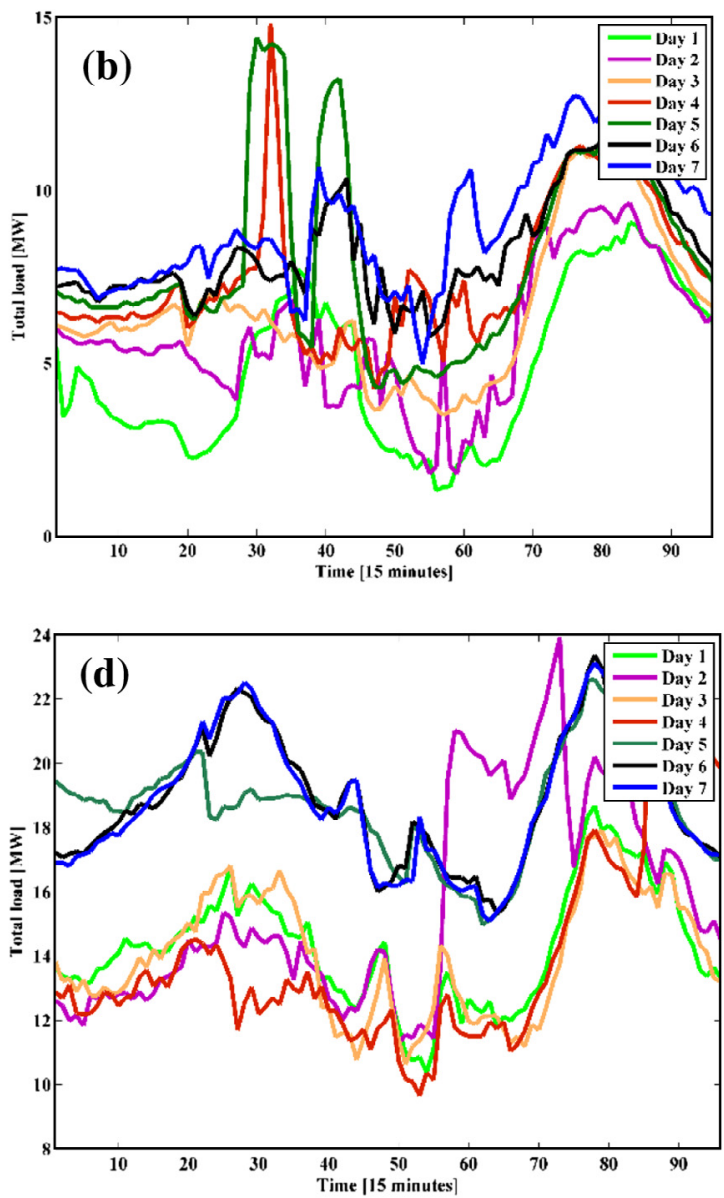

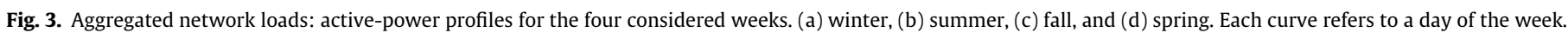


Table 2

OPtimal ESS site and size.

\begin{tabular}{lllll}
\hline ESS \# & 1 & 2 & 3 & 4 \\
\hline Node number & 41 & 159 & 230 & 233 \\
Power rating (MW) & 1.1 & 1.87 & 0.47 & 1.12 \\
Reservoir capacity (MWh) & 3.4 & 3.32 & 0.48 & 1.15 \\
\hline
\end{tabular}

the voltage exhibits a deviation from the rated value larger than $\pm 2 \%$.

The obtained optimal locations and ratings of ESSs are shown in Table 2. As it can be seen, 4 nodes are selected to install ESSs. All the selected nodes are close to the largest loads. For the two cases of the grid without and with optimally located ESSs, Table 3 shows the total amount of network losses, feeder overload above $80 \%$ of the rated current and energy cost imported from the external grid. All these quantities exhibit a clear decrease in case where optimally-allocated ESSs are available in the network.

Fig. 5 shows the Cumulative Distribution Function (CDF) of nodal voltages in both analyzed cases (i.e., with and without ESSs). It is evident that the presence of ESSs allows to largely improve the ADN quality-of-service with respect to voltage variations.

The SoC of all the ESSs in two days, one day in summer and one day in winter, are shown in Figs. 6 and 7 respectively. As it can be observed, the figures show how the integral constraint on the ESS SoC has been respected. Figs. 6 shows that, in the summer period, all of the ESSs, except the one that is on the feeder with PV production follow the load profile. The ESS 1, which is located on the feeder with PV production is responding to the energy price profile. It stores excess PV production during the day and produce energy during high-peak hours. Fig. 7 shows that the ESSs SoC in the wintertime period is different. In particular, all the ESSs are responding to the load profile. In this respect, it is worth observing that these ESSs are located in the feeders characterized by the highest loading level with associated largest voltage variations. Thus, they tend to minimize the corresponding elements of the multi-objective function since they have a larger priority. In view of the above considerations, it is evident how the proposed process is capable to locate each ESS by distinguishing their influences on: the network quality-of-service, the local energy balance and the network zone of influence.

It should be noted that the case study investigated in this paper does not require any load curtailment. It is also worth noting that the main objective investigated in this paper is the ESSs contribution to increase the ADN quality-of-service (i.e., compensate the voltage deviations). Therefore, this specific objective is characterized by the highest weight in the objective function. As a result, as it can be noted from Table 3, the benefits resulting from the energy arbitrage cannot justify the ESS high capital cost alone.

A reasonable stopping criterion is that the primal and dual residuals have to be small [18]. The objective sub-optimality is small when these two values are small [18]. The progress of primal and dual residuals as a function of the iteration number is shown in Figs. 8 and 9, respectively. The primal and dual residuals are calculated as (19) and (20) respectively and used as the stopping criteria [18].

Table 3

Changes in the each term of the objective function.

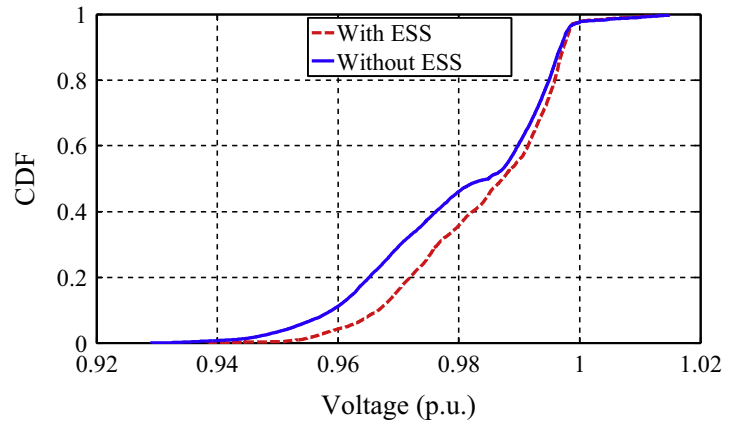

Fig. 5. The CDF of nodal voltages for the cases with and without optimal ESS siting and sizing.

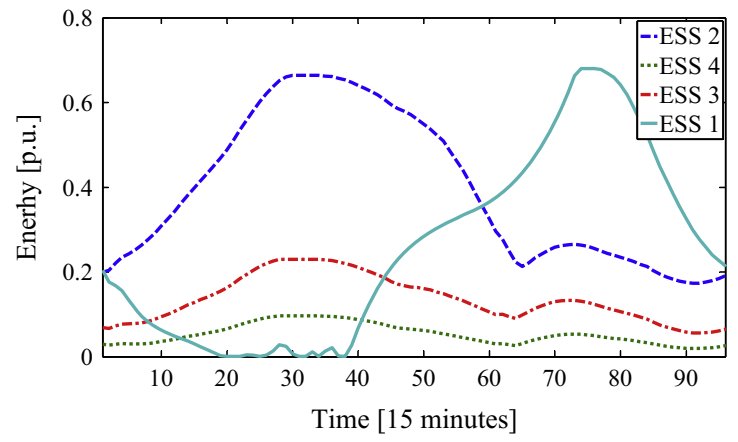

Fig. 6. SoC profiles of the ESSs in summer period (base value of energy is $5 \mathrm{MW} \mathrm{h}$ )

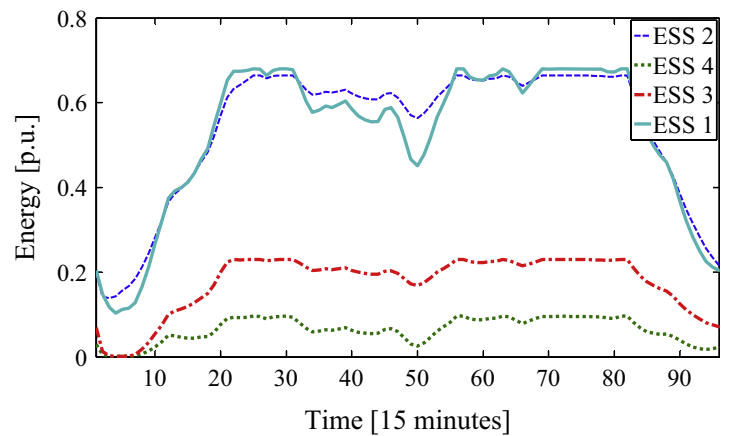

Fig. 7. SoC profiles of the ESSs in winter period (base value of energy is $5 \mathrm{MW} \mathrm{h}$ ).

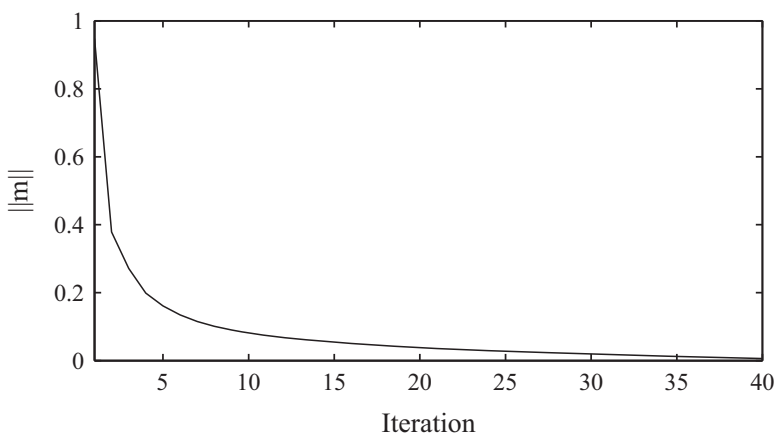

Fig. 8. Norm of primal residual versus iteration.

\begin{tabular}{|c|c|c|c|}
\hline & $\begin{array}{l}\text { Total network losses in } \\
\text { the simulated weeks (MWh) }\end{array}$ & $\begin{array}{l}\text { Total energy cost imported from } \\
\text { the external grid in the simulated weeks (CHF) }\end{array}$ & $\begin{array}{l}\text { Total feeders loading with } \\
\text { percentages larger than } 80 \% \text { (p.u.) }\end{array}$ \\
\hline Optimal ESS siting and sizing & 714.7 & 898450 & 0 \\
\hline Without ESS & 859.02 & 985140 & 14.7 \\
\hline
\end{tabular}




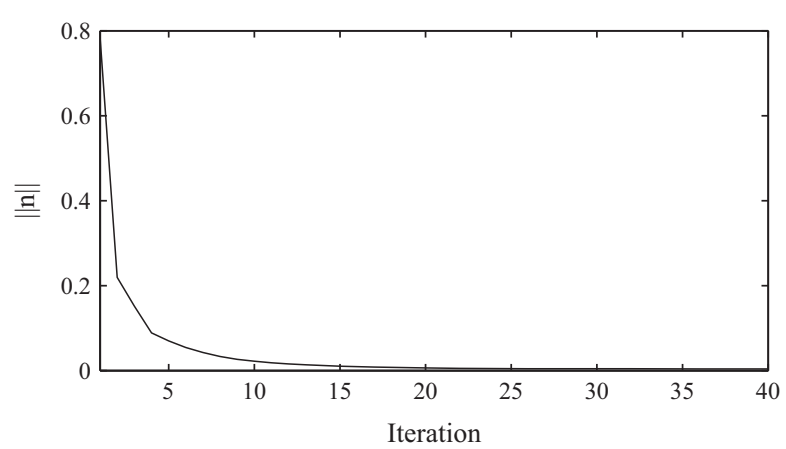

Fig. 9. Norm of dual residual versus iteration.

Table 4

Computational time comparison between two cases: (i) with ADMM, (ii) without ADMM.

\begin{tabular}{clll}
\hline & 1 scenario & 10 scenarios & 15 scenarios \\
\hline ADMM & $43 \mathrm{~s}$ (converged in & $41 \mathrm{~s}$ (convergence & $42 \mathrm{~s}$ (convergence \\
& 40 iterations) & in 40 iterations) & in 40 iterations) \\
Without & $3680 \mathrm{~s}$ & $35952.98 \mathrm{~s}$ & $50772.87 \mathrm{~s}$ \\
ADMM & & & \\
\hline
\end{tabular}

$\left\|m^{k}\right\|=\left\|A x^{k}+B z^{k}-c\right\|$

$\left\|n^{k}\right\|=\left\|z^{k+1}-z^{k}\right\|$

\section{Computational performances}

This section illustrates the computational advantages associated to the use of the ADMM with respect to the case in which we do not decompose it. In this respect, Table 4 shows the computational time to solve the problem with respect to these two conditions.

As it can be inferred from Table 4, with the increase of the number of scenarios, the computational time increases almost exponentially for the case of a solution without the ADMM. Additionally, when the number of scenarios increase, also the memory allocation required by the solver increases causing outof-memory issues. On the other hand, with ADMM the increase of scenarios just increase the number of sub-problem and it does not increase the size of each individual optimization subproblem.

It should be also noted that the value of penalty parameter $\rho$ has an important impact on the computational time of the subproblems and the number of iterations required for the convergence. With a small values of $\rho$ (i.e., 0.5 ), the computational time of each subproblem at the first couple of iteration is around $400 \mathrm{~s}$ and the required number of iterations for convergence is around 100 whereas with $\rho$ equal to 100 the computational time is around $40 \mathrm{~s}$ and the problem converges in less than 40 iterations.

\section{Conclusion}

The paper has proposed a decomposition method based on the ADMM methodology applied to the problem of ESSs optimal siting and sizing in ADNs. The objective function of the problem accounts for different benefits of the ESSs. Indeed, the targeted problem has been formulated to account for: voltage deviations, network losses, in addition to investment and operation cost minimizations and congestion management.
Compared to other works already published by the Authors on the same subject, the paper has shown how the use of ADMM has allowed to define an efficient procedure to solve a large-scale problem accounting for networks with realistic large number of nodes and scenarios. In this respect, after the description of the proposed planning procedure, the paper has discussed its application to the case of a real large-scale network with real profiles of load and distributed generation coming from photovoltaic units.

It is evident from the obtained results how the proposed process is capable to locate each ESS by distinguishing their influences on: the network quality-of-service, the local energy balance and the network zone of influence. It can be concluded that the proposed process can be used by DNOs to evaluate the possible use of ESSs as a valid alternative to investments in grid reinforcement or massive telecom infrastructure for direct DG control.

\section{References}

[1] Cigre TB 458. Electric Energy Storage Systems. CIGRE Publication, April 2011.

[2] Oudalov A, Cherkaoui R, Beguin A. Sizing and optimal operation of battery energy storage system for peak shaving application. In: Lausanne Powertech conference, July 2007, p. 1-5.

[3] Barton JP, Infield DG. Energy storage and its use with intermittent renewable energy. IEEE Trans Energy Conv 2004;19(2):441-8.

[4] Nick M, Hohmman M, Cherkaoui R, Paolone M. On the optimal placement of distributed storage systems for voltage control in active distribution networks. In: Proc of the IEEE int conf on Smart Grids Technologies (ISGT) Europe, October 2012. p. 1-6.

[5] Mercier P, Cherkaoui R, Oudalov A. Optimizing a battery energy storage system for frequency control application in an isolated power system. IEEE Trans Power Syst 2009;24(3):1469-77.

[6] Celli G, Mocci S, Pilo F, Loddo M. Optimal integration of energy storage in distribution networks. IEEE PowerTech 2009(7):1-7.

[7] Chen C, Duan S, Cai T, Liu B, Hu G. Optimal allocation and economic analysis of energy storage system in microgrids. IEEE Trans Power Elec 2011;26(10): 2662-773.

[8] Changsong C, Duan S, Cai T, Liu B, Hu G. Optimal allocation and economic analysis of energy storage system in microgrids. IEEE Trans Power Elec 2011; 26(10):2762-73.

[9] Atwa M, El-Saadany E. Optimal allocation of ESS in distribution systems with a high penetration of wind energy. IEEE Trans Power Syst 2010;25(4):1815-22.

[10] Carpinelli G, Mottola F, Proto D, Russo A. Optimal allocation of dispersed generators, capacitors and distributed energy storage systems in distribution networks modern electric power systems conference, September 2010, p. 1-6.

[11] Zheng Yu, Dong Zhao Yang, Luo Feng Ji, Meng Ke, Qiu Jing, Wong Kit Po. Optimal allocation of energy storage system for risk mitigation of DISCOs with high renewable penetrations. IEEE Trans Power Syst 2014:29(1):212-20.

[12] Oh H. Optimal planning to include storage devices in power systems. IEEE Trans Power Syst 2011;26(3):1118-28.

[13] Fallahi F, Nick M, Riahy GH, Hosseinian SH, Doroudi A. The value of energy storage in optimal non-firm wind capacity connection to power systems. Renewable Energy 2014;64:34-42.

[14] Nick M., Hohmman M, Cherkaoui R, Paolone M. Optimal location and sizing of distributed storage systems in active distribution networks. In: 10th PowerTech conference, Grenoble, June 2013, p. 1-6.

[15] Nick M, Cherkaoui R, Paolone M. Optimal allocation of dispersed energy storage systems in active distribution networks for energy balance and grid support. IEEE Trans Power Syst 2014;29(5):2300-10.

[16] Dall'Anese E, Zhu H, Giannakis GB. Distributed optimal power flow for smart microgrids. IEEE Trans Smart grid 2013;4(3):1464-75.

[17] Shahidehopour M, Fu Y. Benders decomposition: applying Benders decomposition to power systems. IEEE Power Energ Mag 2005;3(2):20-1.

[18] Boyd S, Parikh N, Chu E, Peleato B, Eckstein E. Distributed optimization and statistical learning via the alternating direction method of multipliers. Found Trends ${ }^{\circledR}$ Mach Learn 2011;3(1):1-122.

[19] Fukushima M. Application of the alternating direction method of multipliers to separable convex programming problems. Comput Opt Appl 1992;1(1): 93-111.

[20] Gan L, Li N, Topcu U, Low S. Exact convex relaxation of optimal power flow in radial networks. arXiv preprint arXiv 2012.

[21] Nick M, Cherkaoui R, Paolone M. Optimal siting and sizing of distributed energy storage systems via alternating direction method of multipliers. 18th Power Systems Computation Conference (PSCC), Wroclaw, August 2014.

[22] Saaty T. Decision making - the Analytic Hierarchy and Network Processes (AHP/ANP). J Syst Sci Syst Eng 2004;13(1):1-35.

[23] Rastler D. Electricity energy storage technology options: a white paper primer on applications, costs and benefits. Electric Power Research Institute, December 2010. 